Molecular Brain

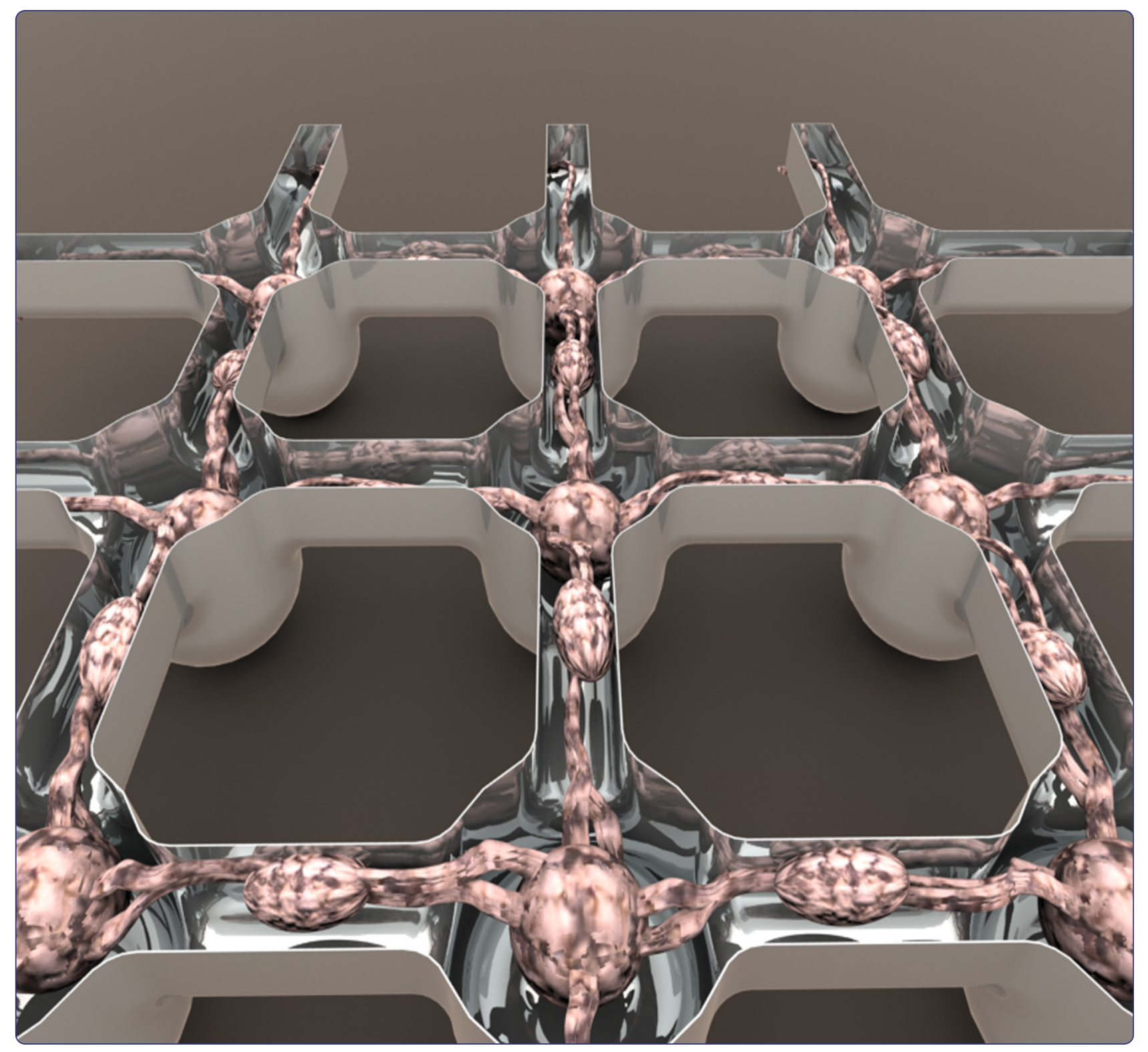

\title{
Networked neural spheroid by neuro-bundle mimicking nervous system created by topology effect
}

Jeong et al.

() Biomed Central 


\title{
Networked neural spheroid by neuro-bundle mimicking nervous system created by topology effect
}

\author{
Gi Seok Jeong ${ }^{1}$, Joon Young Chang ${ }^{1}$, Ji Soo Park ${ }^{1,4}$, Seung-A Lee ${ }^{1}$, DoYeun Park' ${ }^{1}$ Junsung Woo ${ }^{2,3}$, Heeyoung An ${ }^{2,4}$, \\ C Justin Lee $2,3,4$ and Sang-Hoon Lee ${ }^{1,4^{*}}$
}

\begin{abstract}
In most animals, the nervous system consists of the central nervous system (CNS) and the peripheral nervous system (PNS), the latter of which connects the CNS to all parts of the body. Damage and/or malfunction of the nervous system causes serious pathologies, including neurodegenerative disorders, spinal cord injury, and Alzheimer's disease. Thus, not surprising, considerable research effort, both in vivo and in vitro, has been devoted to studying the nervous system and signal transmission through it. However, conventional in vitro cell culture systems do not enable control over diverse aspects of the neural microenvironment. Moreover, formation of certain nervous system growth patterns in vitro remains a challenge. In this study, we developed a deep hemispherical, microchannel-networked, concave array system and applied it to generate three-dimensional nerve-like neural bundles. The deep hemicylindrical channel network was easily fabricated by exploiting the meniscus induced by the surface tension of a liquid poly(dimethylsiloxane) (PDMS) prepolymer. Neurospheroids spontaneously aggregated in each deep concave microwell and were networked to neighboring spheroids through the deep hemicylindrical channel. Notably, two types of satellite spheroids also formed in deep hemispherical microchannels through self-aggregation and acted as an anchoring point to enhance formation of nerve-like networks with neighboring spheroids. During neural-network formation, neural progenitor cells successfully differentiated into glial and neuronal cells. These cells secreted laminin, forming an extracellular matrix around the host and satellite spheroids. Electrical stimuli were transmitted between networked neurospheroids in the resulting nerve-like neural bundle, as detected by imaging $\mathrm{Ca}^{2+}$ signals in responding cells.
\end{abstract}

Keywords: Neurospheroid, Neural spheroid networking, Deep hemicylindrical channel, Neural bundle, Nerve-like structure

\section{Background}

The nervous system in an animal transmits signals between each organ and the brain, serving to coordinate voluntary and involuntary activities [1-3]. In most animals, the nervous system consists of the central nervous system (CNS) and peripheral nervous system (PNS), the latter of which connects the CNS to all parts of the body [3-5]. Damage and/or malfunction of the nervous system causes serious pathologies, including neurodegenerative disorders,

\footnotetext{
* Correspondence: dbiomed@korea.ac.kr

'Department of Biomedical Engineering, College of Health Science, Korea University, Seoul 136-100, South Korea

${ }^{4}$ KU-KIST Graduate School of Converging Science and Technology, Korea University, Seoul 136-701, South Korea

Full list of author information is available at the end of the article
}

spinal cord injury, and Alzheimer's disease. Given its prominent functional role, the nervous system has been the continuing focus of extensive studies.

One aspect of nervous system function that attracted considerable attention is signal transmission through the system. Signals within the nervous system are transmitted by an electrochemical wave called an action potential, which travels along the nerves, composed of cylindrical bundles of fibers consisting mostly of neural axons. The signal is transmitted between nerves by small amounts of neurotransmitter molecules released at nerve junctions, termed synapses. A variety of in vitro approaches have been developed in an attempt to understand the signal transduction mechanisms of this critically important system. However, conventional in vitro cell culture plates 
do not provide the ability to control diverse features of the neural microenvironment, and formation of certain neuronal growth patterns that mimic those that occur in vivo remains a challenge [6-8]. Recent progress in microfluidics, including micro contact printing and micro- and nano-topology fabrication technology, have allowed the culture of neuronal cells in a well-defined microenvironment, enabling the control of neuron and glial cell structuring processes. Microscale chemical $[9,10]$ and topological patterns have proven invaluable for the study of neuronal behavior. Representative examples of these techniques include gradient control of soluble biochemical cues [11-14], micro-engineered grooved patterns $[15,16]$, and biochemically modified grooved substrates $[17,18]$. These methods have been used extensively for guiding the growth of neurons $[8,15,16,19]$ and promoting neuro-networking $[7,20]$. Although these approaches yield well-defined, networked neural cultures, it remains difficult to create a neural functionality close to that of the in vivo environment because cell culture conditions are restricted to a two-dimensional (2D) surface. Three-dimensional (3D) neuro-spheroid and -bundle formation of the nervous system by culturing on controlled microstructures, which have been shown to support successful growth of neurites, have been proposed as an alternative for mimicking the in vivo microenvironment [14,21,22].

Although $2 \mathrm{D}$ and $3 \mathrm{D}$ formation in in vitro nervous systems facilitate neurite growth and networking, most such models are based on the growth of single neurons or a single cell-cell network $[7,8,13,18]$ using primary neuro progenitor cells. However, the nervous system in the animal is created by the growth of multiple cell types, including neurons and glia, which provide structural and metabolic support. In addition, conventional neural cell culture methods have a limited ability to mimic the connections of the nervous system between one part of the body and another through fiber bundles. To this end, several studies have been performed to fabricate three-dimensional (3D) neural networks using a microwell array [7,21-23]. Although, these studies demonstrated successful formation of spheroids and neural networking, it is still challenging to create a bundle-like neural networking formation which mimics the nervous system between spheroids. To address this limit, we have demonstrated that topological factors are critical for the formation of a nervous system, and further showed that a hemicylindrical channel is more effective in guiding neural outgrowth than a rectangular channel [24]. However, the neural bundle in this system was weakly connected through the hemicylindrical channel, and it was difficult to observe signal transmission through the bundle. For improved formation of bundle-like structures, we discovered that the channel barrier plays an important role in guiding well-defined outgrowth of multiple neurites, and quantitative studies for the barrier effect for the nervous system formation were required. In this study, we demonstrate a 3D nervous system model in which neurospheroids are networked to neighboring neurospheroids by a nerve-like network through deep hemicylindrical channels. To fabricate deep hemicylindrical channels, we introduced a method for extracting remnant poly(dimethylsiloxane) (PDMS) prepolymer from microwells and channels. The resulting concave wells supported the self-aggregation of host neurospheroids, and the deep hemicylindrical channel between concave wells provided excellent guidance of neural growth. Unlike the case for shallow hemicylindrical channels, satellite neurospheroids formed in the deep hemicylindrical channel. These satellite spheroids, guided by the deep hemicylindrical channel, played a bridging role in forming networks between host spheroids, enhancing the formation of strong nerve-like structures. To confirm this topological effect on the formation of nerve-like structures, we conducted a series of experiments comparing deep hemicylindrical channel well networking $(\mathrm{HCWN})$ systems with shallow HCWN systems. These experiments demonstrated successful differentiation of neural progenitor cells to glia and neurons in the deep HCWN system. Moreover, subsequent $\mathrm{Ca}^{2+}$ imaging revealed propagation of electrical stimuli between networked host spheroids, confirming formation of functional nerve-like networks. Laminin membranes also developed around host and satellite neurospheroids during the process of neural progenitor cell differentiation. The proposed 3D nervous system could be extended to serve as a model for pathophysiological studies of the nervous system as well as nervous system diseases.

\section{Results}

\section{Formation of deep HCWN features through PDMS surface tension}

Using a sweeping and suctioning process, we successfully fabricated shallow and deep hemicylindrical channels, and concave microwells from the PDMS base mold without the use of complicated devices or processes. For fabrication of shallow HCWN plates, only a sweeping process was employed, resulting in the generation of a shallow ( $\sim 0 \mu \mathrm{m}$ deep) hemicylindrical channel. The residual PDMS polymer was approximately $41.97 \pm 3.57 \mathrm{mg}$ (after sweeping) and $33.91 \pm 2.75 \mathrm{mg}$ (after suction) in the base mold (Additional file 1: Figure S1e). Figure 1b shows a side view of an SEM image of the shallow hemicylindrical channel and concave wells integrated in the HCWN system. Deep HCWN plates were prepared similarly, but with addition of a suctioning step to remove remnant PDMS polymer. After suctioning, the height of the hemicylindrical channel was almost the same as the 


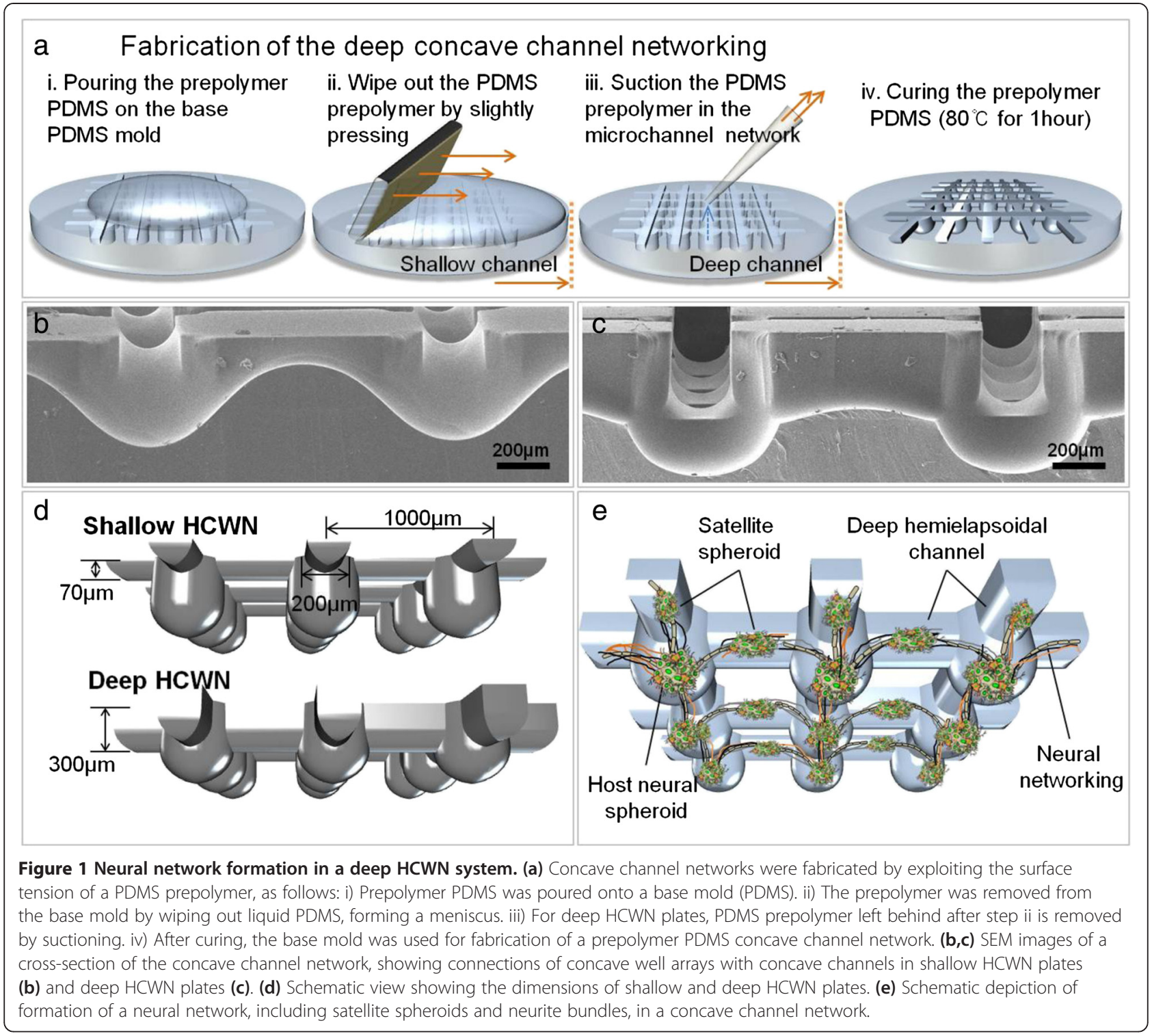

rectangular channel height $(\sim 300 \mu \mathrm{m})$ of the PDMS base mold (Figure 1c). Figure 1d shows an illustration of the shape and dimensions of shallow and deep HCWN plates. As shown in the figures, the deeper hemicylindrical channel is suitable for generating satellite spheroids owing to the comparatively smooth joint of the deep channel (Figure 1e).

\section{Topological effects of shallow and deep HCWN systems on neural network formation}

To confirm the topological effect of the deep hemicylindrical channel in guiding neural cells, we performed comparison studies with shallow and deep HCWN systems. Networked neurospheroids were generated by first seeding both platforms with primary rat fetal cortical neural progenitor cells. The cells that did not settle in concave wells or hemicylindrical channels were removed by gentle pipetting (Additional file 1: Figure S3a). As a result, the seeded cells mainly remained in the concave microwells and deep hemicylindrical channels (Additional file 1: Figure S3b and c). We hypothesized that the residual cells in the deep hemicylindrical channel could play a critical role in fostering strong networking within neural bundles (Additional file 1: Figure S3c). Figure $2 \mathrm{a}$ and $\mathrm{b}$ show a schematic of the neural networking process in shallow and deep HCWN systems. As expected, neural cells in the shallow HCWN system only self-aggregated within the concave wells, forming neurospheroids (Figure 2c, white arrowheads); neural networks between spheroids formed via the shallow hemicylindrical channel (Figure 2c, red arrowheads), but satellite spheroids in channels were not observed. 


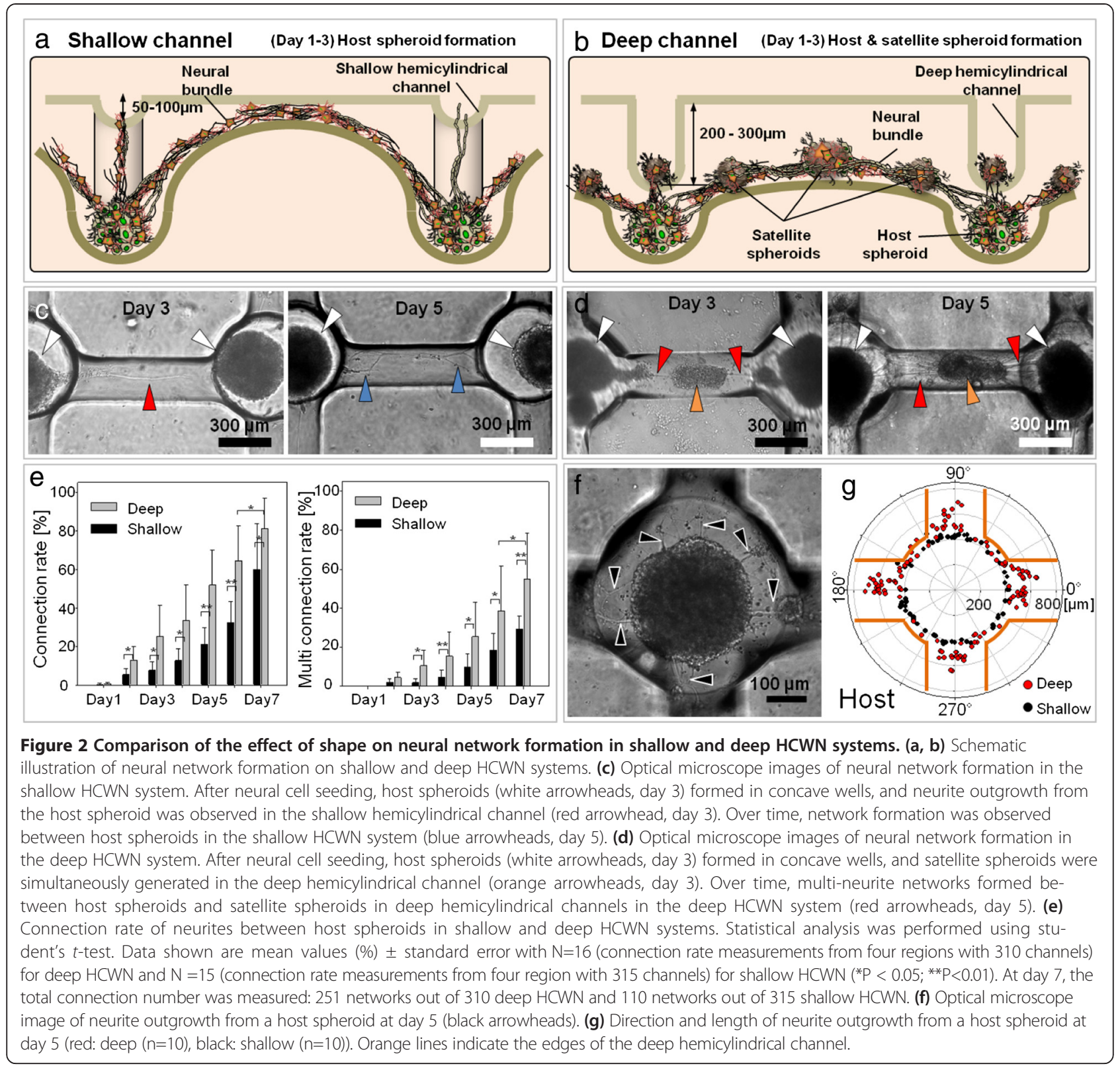

Neural cells seeded in deep HCWN plates also formed well-aggregated spheroids in concave microwells, but interestingly, the residual cells in the deep hemicylindrical channel generated satellite spheroids (Figure 2d, day 3, orange arrowheads). In the deep HCWN system, the neural network (red arrowheads) connected host (white arrowheads) and satellite (orange arrowheads) spheroids. About 5 days after seeding, neural networks between host and satellite spheroids were connected by multiple neurite strands growing along the deep hemicylindrical channel (Figure 2d, red arrowheads), with satellite spheroids acting as bridges during neural bundle formation (Figure 2b and d, red arrowheads). To quantify these results, we determined neural network connection rates, including overall rate and single and multiple connection rates, as defined in Additional file 1: Figure S4. These results, presented in Figure 2e, indicate that single, multiple, and bundle-formation connection rates were approximately $20 \%$ faster for the deep HCWN system (Figure 2e, left) at a given day in culture compared to the shallow HCWN system. About $80 \%$ of neurites were networked after 7 day in culture in the deep HCWN system. In contrast, approximately $60 \%$ of neurites were connected in the shallow HCWN system at this same time point (Figure 2e, left). To examine formation of nerve-like structures, we quantified multiconnections and bundles. Multi-connections in neural networks, defined as cases in which multiple connections 
or neural bundles formed along the channel (Additional file 1: Figure S4b), also increased over time. Seven days after cell seeding, the deep HCWN system achieved a multi-connection rate of about $60 \%$; in contrast, the multi-connection rate in the shallow HCWN system at this time was about $30 \%$. We further assessed neurite outgrowth from host spheroids in deep HCWN plates (Figure 2f), and quantified their length and direction of outgrowth (Figure 2g). Neurite outgrowth from host spheroids was mainly $(\sim 80 \%)$ connected along the hemicylindrical channel, indicating that the deep hemicylindrical channel has a strong affinity for neurites, providing guidance cues and directional control of neurite growth. In shallow HCWN plates, neurites frequently grew outside of the channel (Additional file 1: Figure S5).
Topological effects of the deep HCWN system on satellite spheroid formation

As noted above, satellite spheroids in the deep HCWN system enhanced neural network formation by serving an anchoring role between host spheroids. Our experiments showed two main types of satellite formation in the deep hemicylindrical channel, reflecting the nature of the satellite spheroid formation process. As shown in Figure 3a (i-iii), after the cell-seeding and wash-out step, neural cells settle down onto the concave microwells and deep hemicylindrical channel. One days after cell seeding, the host spheroids were self-aggregated in the concave microwell (host spheroid), and a number of neurite migrate out from the host spheroid, spontaneously generating type I satellite spheroids at the interfacing region between the concave microwells and hemispherical channel (Figure $3 \mathrm{~b}$ and $\mathrm{c}$, red
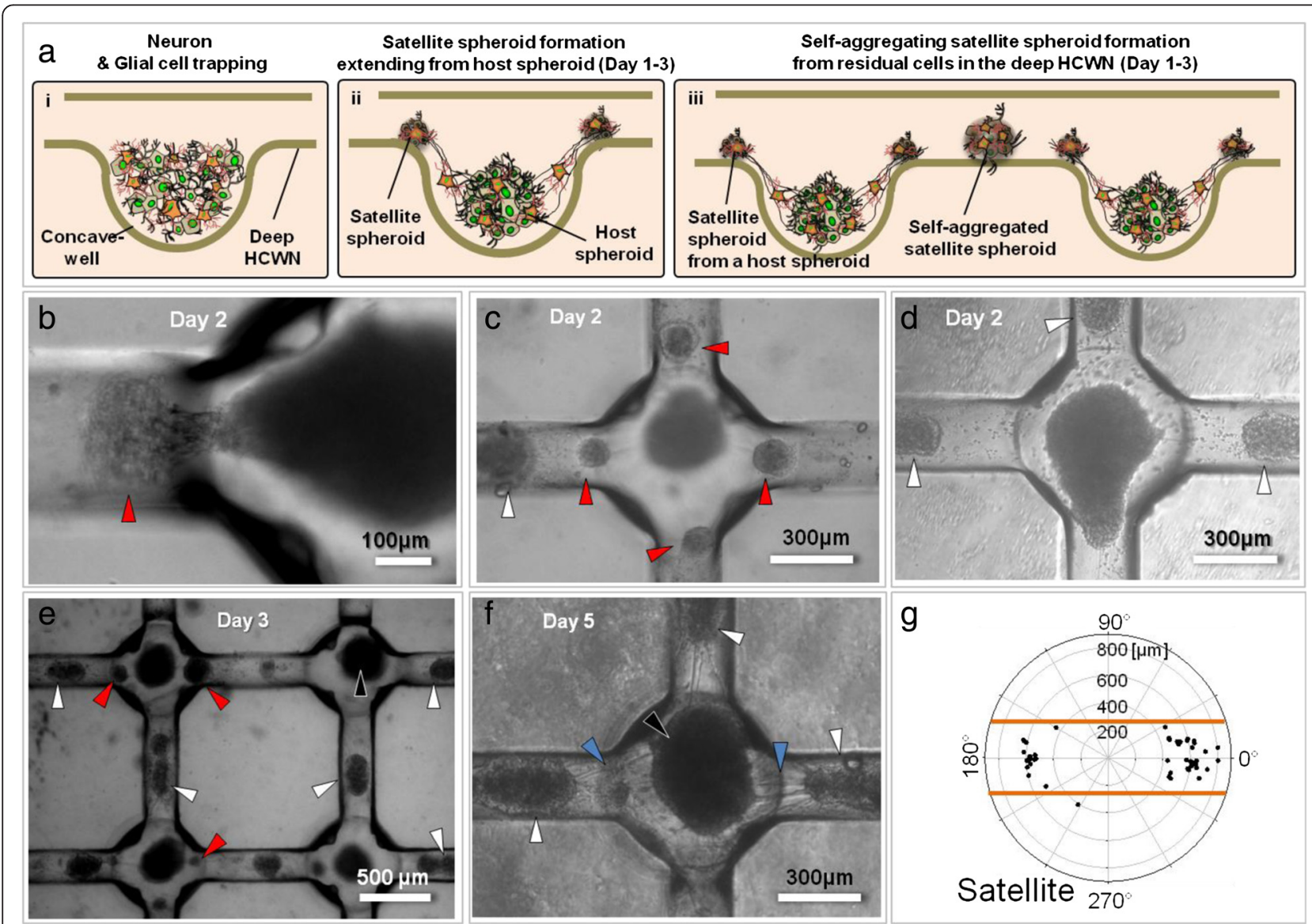

Figure 3 Satellite spheroid formation in deep hemicylindrical channels. (a) Schematic illustration of neural cell seeding (i) and satellite spheroid formation (ii-iii) on the deep HCWN system. Type I satellite spheroids are generated from the host spheroid (ii), whereas type II spheroids are generated by self-aggregation of residual cells (iii). (b) An optical microscope image of a satellite spheroid formed by migration from a host spheroid (type I, red arrowhead). (c) Satellite spheroids in the center of deep hemicylindrical channels spontaneously self-aggregated from residual cells (type II, white arrowheads). (d) Optical microscope image of type I and type II satellite spheroids formed simultaneously around the host spheroid on deep hemicylindrical channels. (e) Network formation between host spheroids (black arrowheads), type I spheroids (red arrowheads), and type II spheroids (white arrowheads) was observed in the deep HCWN system. (f) Multi-neurite connections between the host spheroid and satellite spheroids (blue arrowheads) in the deep HCWN system. (g) Direction and length of neurite outgrowth from satellite spheroids in a hemicylindrical channel at day 5. Orange lines indicate the edges of the deep hemicylindrical channel. 
arrowheads). Especially, residual cells in the deep hemicylindrical channel (Additional file 1: Figure S3c) were also self-aggregated to form type II satellite spheroids due to flat shape of the deep hemicylindrical channels (Figure 3c and d). Contrary to the shallow HCWN channel, these two types of spheroids, as well as host spheroids, are generated simultaneously throughout the deep HCWN system (Figure 3e). Furthermore, over time, these satellite spheroids merge with each other, contributing to the formation of multi-connections and bundles of nerve-like structures in the deep HCWN system (Figure 3f). Quantification of neurite outgrowth from satellite spheroids in the deep HCWN system (Figure $3 \mathrm{~g}$ ) showed that about 95\% of neurites grew along the deep hemicylindrical channel, demonstrating that topology and satellite spheroids in the deep hemicylindrical channel effectively control neurite outgrowth.

\section{ECM membrane secretion and glial cell differentiation}

In this study, neural networking spheroids were generated by culturing cells without an ECM coating. However, it is well known that neural cells modify their environment by secreting the ECM component laminin [25]. Notably, ECM plays a pivotal role in neural cell guidance, proliferation, migration and differentiation, especially during the neuronal development process. We thus hypothesized that neural cells in the deep HCWN system secreted EMC, as depicted in the schematics shown in Figure 4a and b. Consistent with this possibility, SEM images revealed the presence of a membrane structure in deep HCWN plates 10 days after seeding primary neural cells, especially around host and satellite spheroids (Figure $4 \mathrm{c}$ and d). To verify that this membrane structure is ECM, we immunostained laminin. We found that laminin was strongly expressed around host spheroids (Figure 4e)z. Confocal and fluorescence microscope images of laminin expression

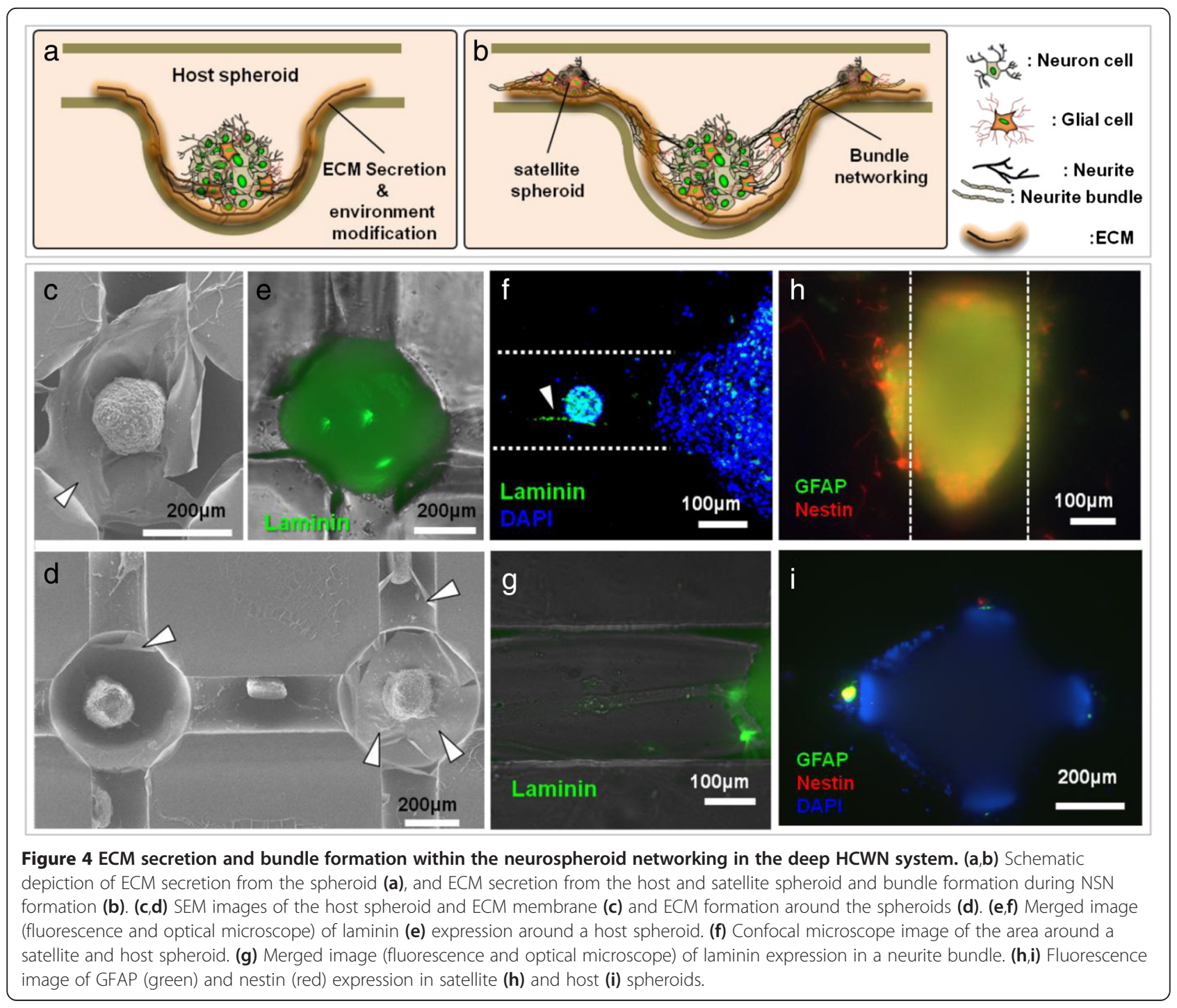


in concave wells and hemicylindrical channels of deep HCWN plates showed that laminin fluorescence was highly expressed on host and satellite spheroids (Figure $4 \mathrm{f}$ and g; bright blue in merged images). Differentiation of progenitor neurons was assessed by immunostaining for the glial cell marker GFAP (glial fibrillary acidic protein) and progenitor cell marker nestin. Both markers were expressed in similar regions on satellite spheroids in the deep HCWN system (Figure 4h), particularly around the joint region of host spheroids (Figure 4i).

Neuronal signal transmission through neurite bundles Neural cells were cultured in deep HCWN plates for 7 days to allow the development of neural networks connected through nerve-like bundles (Figure 5a and b). Satellite spheroids (Figure $5 \mathrm{~b}$, white arrowheads) in the deep hemicylindrical channel were anchored at the center of the channel and were connected to the host spheroids (Figure $5 \mathrm{a}$ and $\mathrm{b}$, orange arrowheads), showing that satellite spheroids act as intermediaries to enhance structural networking between host spheroids. To determine whether this nerve-like network supports signal transmission functions, we applied an electrical stimulus to one spheroid (Figure 5c) and recorded $\mathrm{Ca}^{2+}$ responses of fura-2-loaded cells in a connected spheroid. As shown in Figure $5 \mathrm{~d}$, electrical stimulation at one point produced simultaneous $\mathrm{Ca}^{2+}$ fluorescence signals in responding cells located at a distance of $\sim 1 \mathrm{~mm}$ (Figure $5 \mathrm{~d}$, white dotted

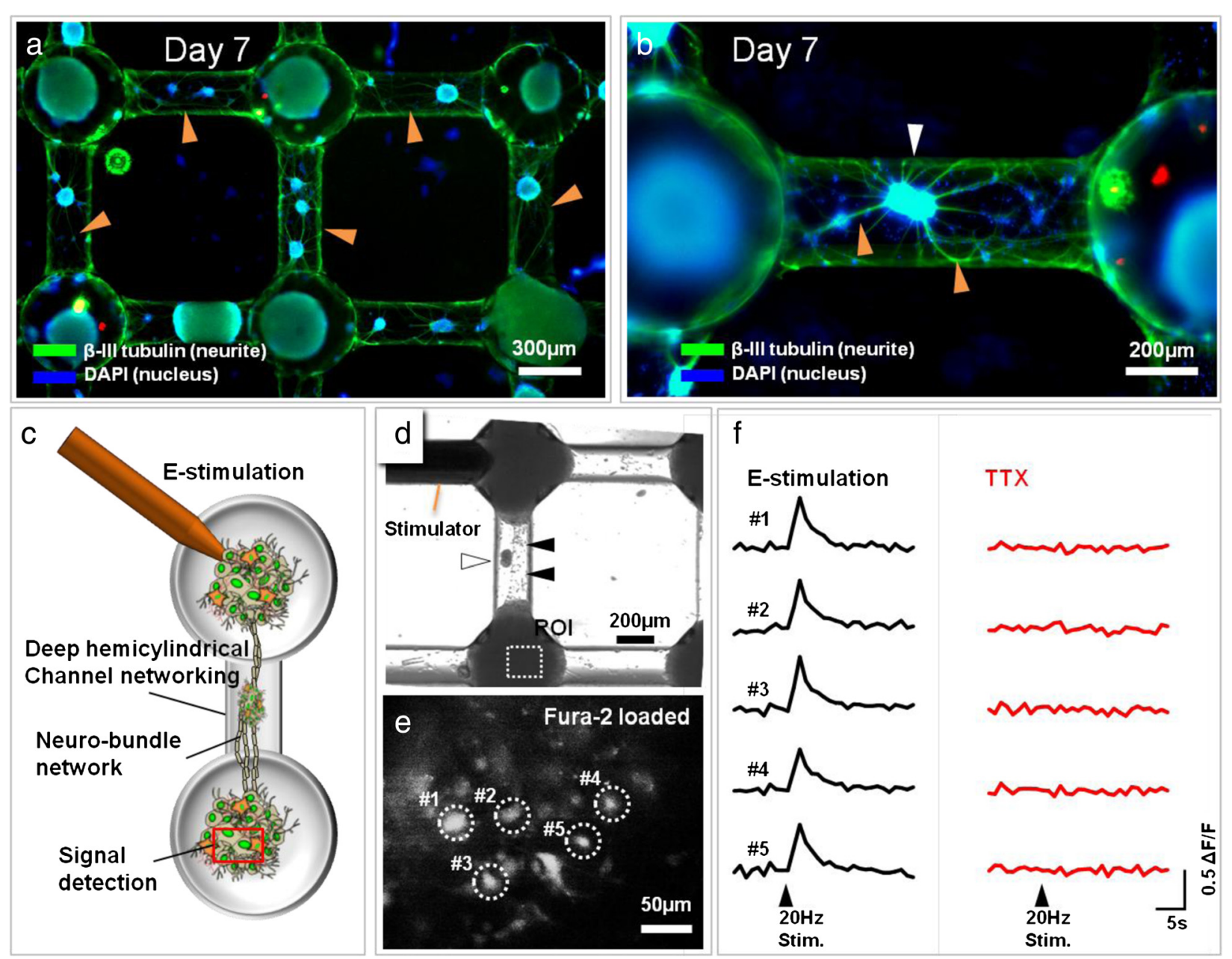

Figure $\mathbf{5}$ Characterization of neural bundle-spheroid network function. (a) Fluorescence image of neuronal spheroids. Green and blue indicate neurites ( $\beta$-tubulin) and nuclei (DAPI), respectively. (b) Bundle formation in neurospheroid networks; each neural bundle is composed of several neurite fibers. (c) Schematic depiction of the method used to assess signal transmission within a neurospheroid network. One neuronal spheroid was electrically stimulated and the electrical signal was detected by imaging Ca2+ in another spheroid. Red rectangle indicates the region of interest (ROI). (d) An optical microscope image of an electric stimulator on the nerve-like bundle and the $\mathrm{ROI}$ at which $\mathrm{Ca} 2+$ signals were recorded. Black arrowheads indicate a neural bundle. (e) A fluorescence microscope image of Ca2+ signals (dotted circles) detected in the ROI shown in (d). The white and black arrowheads indicate satellite spheroid and bundle formation in deep HCWN (f) Electrical transmission through the nerve-like bundle without (left) and with (right) TTX. Signals were detected at all sites prior to TXX treatment, and all signals were eliminated by $\Pi \mathrm{X}$. 
rectangle) from the stimulator, which is indicative of transmission of a $\mathrm{Ca}^{2+}$ signal through a neural bundle (Figure 5e, white dotted circles) and confirming that spheroids are functionally networked. The stimulationevoked $\mathrm{Ca}^{2+}$ response was transient and was abolished by the voltage-gated $\mathrm{Na}^{+}$channel inhibitor tetrodotoxin (TTX; $0.5 \mu \mathrm{M}$ ), indicating that a stimulation-induced action potential could be propagated through the networked bundles, leading to a postsynaptic $\mathrm{Ca}^{2+}$ response at the recording site (Figure 5f). These results show that neural network bundles consist of several neuronal cells and connect spheroid to spheroid.

\section{Discussion}

In a previous study, we compared neural network formation in a shallow hemicylindrical channel to that in a rectangular channel [24]. Although the nerve-like network in the shallow hemicylindrical microchannel was generated randomly and was weak, we found that the hemicylindrical channel provided suitable conditions for neurite outgrowth and networking. In the current study, we compared nervous system formation in shallow and deep hemicylindrical channels to observe the barrier effect. Our results demonstrated that the deep hemicylindrical channel exerts a strong topological influence over the generation of satellite neurospheroids, enhancing guidance of neurite outgrowth and creation of a bundle-like nerve system. Cells tended to aggregate at the center of the deep microchannel (Figure 2d, orange arrowheads) forming type 2 spheroids as the shape of the deep channels is flat and presents a high barrier which prevents neuronal outgrowth. The type 1 spheroids generally appeared at the smooth interfacing area between the concave well and hemicylindrical channel (Figure 3b, c, and e, red arrowheads). These two types of satellite spheroids act as anchors to enhance neural network formation in the deep HCWN system. Although further studies of satellite spheroids are needed, such behavior of neural cells in deep HCWN plates seems to be closely related with the topology of the channel. Despite seeding cells on untreated surfaces of deep HCWN plates, neurites grew along the center of the hemicylindrical microchannels even without ECM treatment. These results show that the curvature and depth of the HCWN channels provide a topology which is advantageous for forming a well-guided network of nerves.

Interestingly, the GFAP and Nestin were expressed around the satellite spheroid and at the joint region around the host spheroid, whereas they were not expressed in the host spheroid (Figure $4 \mathrm{~h}$ and i). This figure suggests that some progenitor cells around satellite spheroids and at the joint region are differentiating into glial cells, while cells in host spheroids do not differentiate into glia cells. Although further study is required, this phenomenon seems to reflect the glia cells' role during the nervous system development stage.

Microenvironments have important role for central nerve system (CNS) development and neural stem cell (NSC) differentiation [25], and several ECM molecules have been identified that regulate cell growth, migration and proliferation $[5,16]$, as well as differentiation of neural progenitor cells $[6,8]$. Although, microstructures and biochemical materials can provide cues for growth and differentiation of neuro-progenitor cells, it is still unclear how the neuro-progenitor cells modify the environment to enhance their growth and differentiation. In this study, the deep HCWN seems to enhance the formation of host and satellite spheroids that contributed by secreting laminin, which is well known for playing an important role in neural cell outgrowth and the differentiation of neural progenitor cells [25], and to induce differentiation of the neural cells without any pretreatment of biochemical attractants (Figure 4c, d, and e). The self-modified environment by the secretion of laminin in the deep HCWN can facilitate the formation of well-organized neuro-bundles.

\section{Conclusions}

Using the deep HCWN system, we successfully developed a neural network connecting host and satellite neurospheroids. The host and satellite spheroids created at the deep HCWN structures through self-modification of the surrounding ECM environment. During network formation on the deep hemicylindrical channel network, neural progenitor cells successfully differentiated into glial and neuron cells, forming a laminin-containing scaffold around the host and satellite neurospheroids during nerve-like formation. Functional connectivity within the fabricated nerve-like network was demonstrated by monitoring transmission of an electrical stimulus using $\mathrm{Ca}^{2+}$ imaging. Furthermore, we found that satellite neurospheroids formed around the host neurospheroid on the deep hemicylindrical channel and in the center of the channel. These satellite neurospheroids act as anchoring points in the networks to play an important role in enhancing neural network formation. We expect that the proposed method will be extensively used as a brain or nervous system model for the drug screening and the physiological study.

\section{Methods}

\section{Fabrication of master molds for deep and shallow HCWN systems}

Shallow and deep HCWN plates consist of concave microwell arrays connected by a shallow $(50-100 \mu \mathrm{m})$ or deep $(\sim 300 \mu \mathrm{m})$ hemicylindrical channel. HCWN plates were fabricated by first preparing a two-layered PDMS base mold consisting of cylindrical microwell arrays connected via a rectangular channel using a standard soft lithography process (Additional file 1: Figure S1a). Shallow 
HCWN plates were prepared by pouring PDMS prepolymer on the PDMS base mold (Figure 1a (i-ii) and Additional file 1: Figure S1b), and sweeping out PDMS prepolymer by lightly pressing the soft PDMS base mold using a glass slide $(76 \times 52 \times 1.2 \mathrm{~mm})$, as previously reported [24,26-28] (Figure 1a and Additional file 1: Figure S1b). For deep HCWN plates, the remnant PDMS prepolymer in microwells and channels was aspirated using a syringe (Figure 1a (i-iii) and Additional file 1: Figure S1c), leaving a hemicylindrical channel with a depth of approximately $300 \mu \mathrm{m}$-almost the same depth as the rectangular channel of the PDMS base mold. The residual PDMS prepolymer was measured using a micro balance (PAG214C, OHAUS, USA). For the measurement, the weight of PDMS base mold was measured first. The weight of PDMS base mold after prepolymer sweeping and suction was measured. By subtraction of weight of PDMS base mold, we measured the weight of remaining prepolymer after sweeping and suction. The residual PDMS prepolymer in the microwell and rectangular channel adopted a curved meniscus through surface tension, forming the deep hemicylindrical channels and concave wells. The PDMS prepolymerformed meniscus was solidified by thermal curing on a hot plate $\left(80^{\circ} \mathrm{C}\right.$ for 1 hour) (Figure 1a (iv), and Additional file 1: Figure S1b and c (iv)). After fabrication, shallow and deep HCWN plates were replicated using SU-8 (Micro-Chem, Newton, MA, USA), and a convex SU-8 master mold was created (Additional file 1: Figure S1d and S2). Deep and shallow HCWN plates were ultimately fabricated by replicating the master mold with PDMS. The dimensions of shallow and deep HCWN systems are depicted in Figure 1d. For the deep HCWN system, the dimensions of cylindrical wells were $500 \times 500 \mu \mathrm{m}$ (diameter and depth); rectangular channels were $1 \mathrm{~mm}$ (length) $\times 200 \mu \mathrm{m}$ (width) $\times 300 \mu \mathrm{m}$ (depth) (Additional file 1: Figure S1b and c).

\section{Preparations of neural progenitor cells}

Primary neural progenitor cells were isolated from a cortical region of prenatal (embryonic day 16) rats (DBL, Incheon, South Korea), and were collected by centrifugation at 10,000 rpm for 5 minutes [23,29,30]. After collection, progenitor cells were seeded in wells of deep and shallow HCWN plates and cultured in Neurobasal media (Gibco, Lifetechnonogies, NY, USA) containing B-27 Supplement (Gibco, Lifetechnonogies, NY, USA), $0.5 \mathrm{mM}$ L-glutamine, and $1 \%$ of an antibiotics solution containing 10,000 units penicillin (Gibco, Lifetechnonogies, NY, USA) and streptomycin. All procedures conformed to the standards of the Institutional Review Board of Korea University.

\section{Cell culturing on shallow and deep HCWN plates}

To seed a uniform number of cells into each concave well, we directly dropped $1 \mathrm{ml}$ of a cell suspension $\left(2.0 \times 10^{7}\right.$ cells $\left./ \mathrm{ml}\right)$ on top of each type of HCWN plate followed by repeated gentle pipetting [31,32]. When cells had settled into concave well arrays and channels (10 minutes after seeding), culture medium was gently applied to remove cells that had not settled (Additional file 1: Figure S3). The medium was replaced with fresh medium every other day.

\section{$\mathrm{Ca}^{2+}$ imaging}

Signal transmission between spheroids within formed neural networks was monitored by imaging $\mathrm{Ca}^{2+}$ in responding cells using the ratiometric fluorescent dye, Fura-2-AM (100 $\mu \mathrm{M}$; loading time, 30 minutes). To test whether this $\mathrm{Ca}^{2+}$ response was evoked by neuronal action potential activity, we used tetrodotoxin (TTX), a voltage gated $\mathrm{Na}^{+}$channel inhibitor. To block the neuronal activity, we treated the cells with TTX for at least 5 minutes using a bath application (ACSF recording solution containing TTX). Then, $\mathrm{Ca}^{2+}$ responses were measured upon electrical stimulation. In the presence of TTX, we could not see the $\mathrm{Ca} 2+$ response. The experimental details are now included in the method section. Cells were imaged by exciting at wavelengths of 340 and $380 \mathrm{~nm}$, and collecting fluorescence at $510 \mathrm{~nm}$ using a CCD camera. $\mathrm{Ca}^{2+}$ concentration was determined by performing ratio calculations using Axon Imaging Workbench version 6.2 (Axon Instruments, Molecular Devices, Sunnyvale, CA, USA). To induce the neuronal activity-dependent $\mathrm{Ca}^{2+}$ responses in cells, we used the $20 \mathrm{~Hz}$ electrical stimulation, a subthreshold stimulation. This stimulation has been widely used to induce the neuronal activity in brain slice without causing long-term potentiation [33]. Cells in spheres were electrically stimulated with a $20-\mathrm{Hz}, 1$-second pulse, and images were acquired at a rate of $1 \mathrm{images} / \mathrm{s}$. The relative change in $\mathrm{Ca}^{2+}$ concentration was normalized to baseline concentration, obtained by averaging 20 images prior to stimulation.

\section{Immunochemistry}

For immunostaining, networked neurospheroids were first fixed for 20 minutes with $4 \%$ formaldehyde at $4{ }^{\circ} \mathrm{C}$, after which cells were permeabilized by incubating with phosphate-buffered saline (PBS) containing $0.1 \%$ Triton X-100 for 20 minutes at room temperature, blocked with PBS containing 3\% bovine serum albumen (BSA) for 30 minutes, and then incubated with primary antibody overnight at $4^{\circ} \mathrm{C}$. Neurite outgrowth was examined using a primary rabbit polyclonal antibody against the neurofilament, $\beta$-III tubulin (1:1000; Santa Cruz Biotechnology, Dallas, TX, USA), a marker of neurites. Astrocytes and neural progenitor/stem cells were examined using a primary rabbit polyclonal IgG antibody against GFAP (1:100; Santa Cruz Biotechnology, Dallas, TX, USA) and a primary anti-rat antibody against nestin (1:1000; Stemcell technologies, USA), respectively. After 
incubating overnight, cells were washed with PBS containing $0.1 \%$ BSA for 5 minutes, and then incubated with secondary antibodies (1:1000; Invitrogen, Carlsbad, CA, USA) for 1.5 hours at room temperature. Cells were washed again with $\mathrm{PBS} / 0.1 \% \mathrm{BSA}$, and images were acquired with a fluorescence microscope (EVOS; Advanced Microscopy Group, Mills Creek, WA, USA) after counterstaining with 4,6-diamidino-2-phenylindole dihydrochloride (DAPI; Invitrogen).

For confirmation of laminin, day-10 networked neurospheroids on deep HCWN plates were fixed with $4 \%$ paraformaldehyde for 20 minutes at $4^{\circ} \mathrm{C}$ and permeabilized by incubating with PBS/0.1\% Triton X-100 for 20 minutes at room temperature. Non-specific protein adsorption to cells and membranes of deep HCWN plates was blocked by incubating with $\mathrm{PBS} / 0.1 \%$ BSA for 30 minutes at $4^{\circ} \mathrm{C}$. Cells were then probed with antilaminin (Abcam, Cambridge, UK) overnight at $4{ }^{\circ} \mathrm{C}$. Cells and membranes in deep HCWN plates were gently washed again with $\mathrm{PBS} / 0.1 \%$ BSA and incubated with the appropriate Alexa Fluor 488- or 594- secondary antibody (Invitrogen) for 90 minutes at $4{ }^{\circ} \mathrm{C}$. Fluorescence images were obtained using a confocal laser-scanning microscope (Olympus, Japan).

\section{Scanning electron microscopy}

Concave channel network shape, extracellular matrix (ECM) membrane, and neurospheroid-integrated neural networks in the deep HCWN system were analyzed by field emission-scanning electron microscopy (FE-SEM) using a JEOL $4701 \mathrm{~F}$ system (JEOL Ltd., Tokyo, JAPAN). Neural networks cultured in deep or shallow HCWN plates were first fixed with $2.5 \%$ glutaraldehyde in deionized water for 1 hour, then gently washed with deionized water, and subjected to secondary fixation in $1 \%$ osmium tetroxide in deionized water for 1 hour. Fixed concavechannel neural networks were dehydrated with a series of graded ethanol $(25 \%, 50 \%, 75 \%, 95 \%$, and $100 \%)$, then incubated with tert-butyl alcohol at room temperature for 30 minutes (three times) and frozen at $-70^{\circ} \mathrm{C}$. Neural networks on deep HCWN plates were freeze-dried until the tert-butyl alcohol had evaporated, then were mounted on a specimen stub with graphite tape, coated with palladium alloy, and observed by FE-SEM.

\section{Neurite connection and outgrowth measurements and statistical analysis}

The direction and lengths of neurite outgrowths from host and satellite spheroids were measured in optical microscope images by image analysis using ImageJ (http://rsbweb.nih.gov/ij/). Neurite connections were manually determined from optical microscope images (Additional file 1: Figure S4). The statistical analysis was implemented using SPSS ver.12 (Chicago, IL, USA).

\section{Additional file}

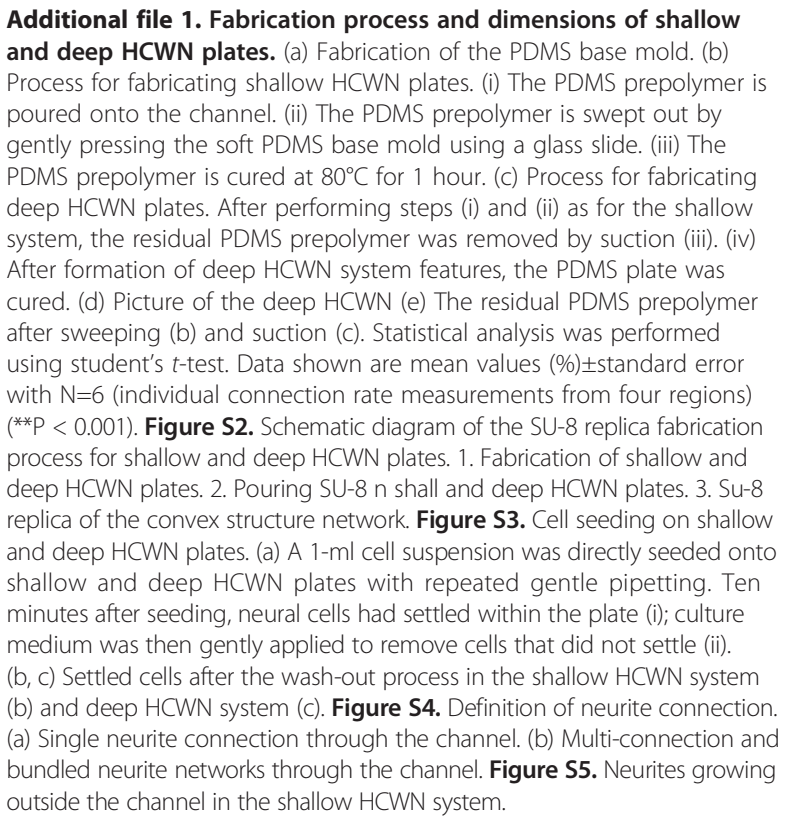

\section{Competing interests}

The authors declare that they have no competing interests.

\section{Authors' contributions}

GSJ designed and carried out the experiments, prepared most of the data, and wrote the manuscript; JYC carried out the experiments and prepared the data; JSP carried out the cell-based evaluation and assisted experiments; DYP assisted and carried out cell based experiments; SL assisted and carried out cell based experiments; JW contributed to prepare data collection; HA assisted to experiments; CJL consulted on the manuscript and contributed in writing the paper; S-HL proposed the idea, managed the research process and wrote the paper. All authors read and approved the final manuscript.

\section{Acknowledgement}

This work was supported by the Industrial Strategic Technology Development Program, the Ministry of Trade, Industry \& Energy (MI, Korea) (Project No.10041923) and Brain Research Program through the National Research Foundation of Korea (NRF) funded by the Ministry of Science, ICT \& Future Planning (2012M3C7A1055410)

\section{Author details}

'Department of Biomedical Engineering, College of Health Science, Korea University, Seoul 136-100, South Korea. ${ }^{2}$ Center for Neural Science and WCI Center for Functional Connectomics, Korea Institute of Science and Technology (KIST), Seoul 136-791, South Korea. ${ }^{3}$ Neuroscience Program, University of Science and Technology (UST), Daejeon 305-350, South Korea. ${ }^{4}$ KU-KIST Graduate School of Converging Science and Technology, Korea University, Seoul 136-701, South Korea.

Received: 14 January 2015 Accepted: 6 March 2015 Published online: 22 March 2015

\section{References}

1. Patrick C. Neural tissue engineering. Ann Biomed Eng. 1997;25(1):p. S-41.

2. Elenkov IJ, Wilder RL, Chrousos GP, Vizi ES, et al. The sympathetic nerve-an integrative interface between two supersystems: the brain and the immune system. Pharmacol Rev. 2000;52(4):595-638.

3. Dayan P, Abbott LF. Theoretical neuroscience. Cambridge, MA: MIT Press; 2001.

4. Maeda E, Robinson HP, Kawana A. The mechanisms of generation and propagation of synchronized bursting in developing networks of cortical neurons. J Neurosci. 1995;15(10):6834-45. 
5. Park J, Kim S, Park SI, Choe Y, Li J, Han A. A microchip for quantitative analysis of CNS axon growth under localized biomolecular treatments. J Neurosci Methods. 2014;221:166-74.

6. Park J, Lim E, Back S, Na H, Park Y, Sun K. Nerve regeneration following spinal cord injury using matrix metalloproteinase-sensitive, hyaluronic acid-based biomimetic hydrogel scaffold containing brain-derived neurotrophic factor. J Biomed Mater Res A. 2009;93(3):1091-9.

7. Kato-Negishi M, Tsuda Y, Onoe H, Takeuchi S. A neurospheroid network-stamping method for neural transplantation to the brain. Biomaterials. 2010;31(34):8939-45.

8. Recknor JB, Sakaguchi DS, Mallapragada SK. Directed growth and selective differentiation of neural progenitor cells on micropatterned polymer substrates. Biomaterials. 2006;27(22):4098-108.

9. Miller RJ, Tran PB. Chemokinetics. Neuron. 2005:47(5):621-3.

10. Gao Y-J, Ji R-R. Chemokines, neuronal-glial interactions, and central processing of neuropathic pain. Pharmacol Ther. 2010;126(1):56-68.

11. Kapur TA, Shoichet MS. Immobilized concentration gradients of nerve growth factor guide neurite outgrowth. J Biomed Mater Res A. 2004;68(2):235-43.

12. Kothapalli $C R$, van Veen $E$, de Valence $S$, Chung $S$, Zervantonakis IK, Gertler FB, et al. A high-throughput microfluidic assay to study neurite response to growth factor gradients. Lab Chip. 2011;11(3):497-507.

13. Park JY, Kim S-K, Woo D-H, Lee E-J, Kim J-H, Lee S-H, et al. Differentiation of neural progenitor cells in a microfluidic chip-generated cytokine gradient. Stem Cells. 2009;27(11):2646-54

14. Choi YJ, Chae S, Kim JH, Barald KF, Park JY, Lee S-H. Neurotoxic amyloid beta oligomeric assemblies recreated in microfluidic platform with interstitial level of slow flow. Sci Rep. 2013;3:1921.

15. Kang E, Jeong GS, Choi YY, Lee KH, Khademhosseini A, Lee S-H. Digitally tunable physicochemical coding of material composition and topography in continuous microfibres. Nat Mater. 2011;10(11):877-83.

16. Kang E, Choi YY, Chae SK, Moon J-H, Chang J-Y, Lee S-H. Microfluidic Spinning of Flat Alginate Fibers with Grooves for Cell-aligning Scaffolds. Adv Mater. 2012:24(31):4271-7.

17. Rhee SW, Taylor AM, Tu CH, Cribbs DH, Cotman CW, Jeon NL. Patterned cell culture inside microfluidic devices. Lab Chip. 2005;5(1):102-7.

18. Baek NS, Kim YH, Han YH, Lee BJ, Kim T-D, Kim S-T, et al. Facile photopatterning of polyfluorene for patterned neuronal networks. Soft Matter. 2011;7(21):10025-31.

19. Yang F, Murugan $R$, Wang S, Ramakrishna S. Electrospinning of nano/micro scale poly (L-lactic acid) aligned fibers and their potential in neural tissue engineering. Biomaterials. 2005;26(15):2603-10.

20. Jun SB, Hynd MR, Dowell-Mesfin N, Smith KL, Turner JN, Shain W, et al. Low-density neuronal networks cultured using patterned poly--lysine on microelectrode arrays. J Neurosci Methods. 2007;160(2):317-26.

21. Choi YJ, Park J, Lee S-H. Size-controllable networked neurospheres as a 3D neuronal tissue model for Alzheimer's disease studies. Biomaterials. 2013;34(12):2938-46.

22. Kato-Negishi M, Morimoto Y, Onoe H, Takeuchi S. Millimeter-Sized Neural Building Blocks for 3D Heterogeneous Neural Network Assembly. Adv Healthc Mater. 2013;2(12):1564-70.

23. Park J, Lee BK, Jeong GS, Hyun JK, Lee CJ, Lee S-H. Three-dimensional brain-on-a-chip with an interstitial level of flow and its application as an in vitro model of Alzheimer's disease. Lab Chip. 2015;15(1):141-50.

24. Jeong GS. Networked neuro-spheres formed by topological attractants for engineering of 3-dimensional nervous system. Tissue Eng Regen Med. 2014;11(4):297-303.

25. Nurcombe V. Laminin in neural development. Pharmacol Ther. 1992;56(2):247-64

26. Jeong GS, Jun Y, Song JH, Shin SH, Lee S-H. Meniscus induced self organization of multiple deep concave wells in a microchannel for embryoid bodies generation. Lab Chip. 2012;12(1):159-66.

27. Jeong GS, Song JH, Kang AR, Jun Y, Kim JH, Chang JY, et al. Surface Tension-mediated, Concave-microwell Arrays for Large-scale, Simultaneous Production of Homogeneously Sized Embryoid Bodies. Adv Healthc Mater. 2013;2(1):119-25.

28. Jeong GS, Lee S-H. Microfluidic spinning of grooved microfiber for guided neuronal cell culture using surface tension mediated grooved round channel. Tissue Eng Regen Med. 2014;11(4):291-6.
29. Lesuisse C, Martin LJ. Long-term culture of mouse cortical neurons as a model for neuronal development, aging, and death. J Neurobiol. 2002;51(1):9-23.

30. Qian X, Shen Q, Goderie SK, He W, Capela A, Davis AA, et al. Timing of CNS cell generation: a programmed sequence of neuron and glial cell production from isolated murine cortical stem cells. Neuron. 2000;28(1):69-80

31. Choi YY, Chung BG, Lee DH, Khademhosseini A, Kim JH, Lee SH. Controlled-size embryoid body formation in concave microwell arrays. Biomaterials. 2010;31(15):4296-303.

32. Wong SF, No DY, Choi YY, Kim DS, Chung BG, Lee S-H. Concave microwell based size-controllable hepatosphere as a three-dimensional liver tissue model. Biomaterials. 2011;32(32):8087-96.

33. MacVicar B, Hochman D. Imaging of synaptically evoked intrinsic optical signals in hippocampal slices. J Neurosci. 1991;11(5):1458-69.

\section{Submit your next manuscript to BioMed Central and take full advantage of:}

- Convenient online submission

- Thorough peer review

- No space constraints or color figure charges

- Immediate publication on acceptance

- Inclusion in PubMed, CAS, Scopus and Google Scholar

- Research which is freely available for redistribution 